\title{
Research on the Performance of Electric Vehicles in Peak Load Shifting
}

\author{
Qingshan Xu, ${ }^{1}$ Yujun Liu, ${ }^{1}$ Maosheng Ding, ${ }^{2}$ Pingliang Zeng, ${ }^{3}$ and Wei Pan ${ }^{1}$ \\ ${ }^{1}$ School of Electrical Engineering, Southeast University, Nanjing 210096, China \\ ${ }^{2}$ State Grid Ningxia Electric Power Company, Yinchuan 750001, China \\ ${ }^{3}$ China Electric Power Research Institute, Beijing 100192, China \\ Correspondence should be addressed to Qingshan Xu; xuqingshan@seu.edu.cn
}

Received 24 January 2014; Revised 6 July 2014; Accepted 10 July 2014; Published 23 July 2014

Academic Editor: Hongjie Jia

Copyright (C) 2014 Qingshan Xu et al. This is an open access article distributed under the Creative Commons Attribution License, which permits unrestricted use, distribution, and reproduction in any medium, provided the original work is properly cited.

\begin{abstract}
Electric vehicles (EVs) are developing remarkably fast these years which makes the technology of vehicle-to-grid (V2G) easier to implement. Peak load shifting (PLS) is an important part of V2G service. A model of EVs' capacity in V2G service is proposed for the research on PLS in this paper. The capacity is valued in accordance with three types of situations. Based on the model, three different scenarios are suggested in order to evaluate the capacity with MATLAB. The evaluation results indicate that EVs can provide potential energy to participate in PLS. Then, the principle of PLS with EVs is researched through the analysis of the relationship between their power and capacity. The performance of EVs in PLS is also simulated. The comparison of two simulation results shows that EVs can fulfill the request of PLS without intensely lowering their capacity level.
\end{abstract}

\section{Introduction}

In recent years, electric vehicles (EVs) are developing rapidly in many countries [1]. Many automotive companies have introduced their new EV products. As a new kind of transportation, EVs have the extraordinary ability to deal with the lack of fuel and the air pollution [2]. Vehicle-to-grid (V2G) is the technology which uses EVs to support the grid and at the same time EVs can obtain power from the grid. Various researches have been done to utilize the EVs to participate in V2G services. V2G services are often applied for wind power balancing [3-7], frequency regulation [8], and peak load shifting (PLS) $[9,10]$.

Among all the hopeful V2G services, PLS is easier to realize and is greatly helpful for the grid. PLS can improve the utilization rate of existing equipment and postpone the upgrade of new equipment. To realize PLS, EVs only have to release part of the energy stored in the EV batteries. There are many researches on the strategy of arranging the EVs' charge and discharge behaviors in order to realize PLS [11, 12]. However, these researches focus on the behavior of every EV and do not research on the essence of PLS with EVs. There are also some researches on evaluating the capacity of EVs [13-15]. But they are not applied for PLS. Besides, there are some researches on PLS with battery storage which has an important reference value $[16,17]$.

This paper researches on the model of EVs' capacity which can be used for PLS. According to the accessing time, all the EVs are divided into three types which have different values of capacity. Then three scenarios are introduced for simulating the specific capacity of EVs. Based on the simulation results, the principle of EVs in PLS is thoroughly analyzed and a few simulations of EVs' performance in PLS are proposed for comparison.

\section{Model of Electric Vehicles' Capacity}

Usually, peak load will last for several hours such as from 19:00 to 21:00. The phenomenon is often caused by the rise of residential load because most people have arrived home at that time. And peak load will go down as night falls. So this characteristic of peak load requests long-time active power support. In order to shift peak load, EVs should be capable of maintaining certain amount of capacity during 
the whole time. But the problem is how much capacity can be achieved from all the EVs connected with the grid at urgent time. Unlike the traditional generators or energy storages, the capacity of EVs is strong stochastic and changeable, because in a short period of time some EVs could leave and some could be connected with the grid.

All the energy stored in the EVs can be discharged for load shifting if the requests of EVs' customers are neglected. But customers' feelings should be respected because they are the people who finally make the decision whether to purchase and drive EVs. At least, EVs should be finished charging at the time their customers want to drive them. Assume that every EV user will set up leaving time $T_{\text {leave }}$ while his EV is plugged in at $T_{\text {arrive }}$ and the EV will leave exactly at that time. In the following studies, it is found that the assumption is reasonable except for emergency use. Most of the EVs will not be so overdischarged for PLS that they cannot deal with a temporary use for short travel. Otherwise the customer ought to choose other transportation instead.

Power batteries are the only equipment to store energy for EVs. State of charge (SOC) is usually used to describe the energy left in the battery. $Q_{x}$ is defined to represent SOC of EV's battery at time $T_{x}$. Different users will request different $Q_{\text {leave }}$. The requirement is related to travel plan of $\mathrm{EV}$ users in the next day. For simplicity of calculation, it is assumed that all users need a fully charged battery at $T_{\text {leave }}$. The assumption will actually increase the requirement for charging and at the same time decrease the capability of PLS. So

$$
Q_{\text {leave }}=1 \text {. }
$$

Now with the assumptions above, the EVs' aggregator knows how long the EV will stay for charging and it could manage all the charge and discharge strategies. At one time period, there are three types of EVs that the aggregator may deal with: accessing time is

(1) so short that EV cannot be fully charged;

(2) enough for discharging all the energy left and then charging until the battery is full;

(3) others.

Actually the accessing time is not the only variable which decides the types. SOC decides the time of discharging all the energy in EV's battery which indirectly judges whether accessing time is enough or not.

All the energy stored in EVs' batteries at time $T_{\text {now }}$ is calculated by

$$
S_{\text {all }}=\sum_{i} \xi_{i} S_{i} Q_{i}
$$

where $S_{\text {all }}$ is the energy of all the EVs at time $T_{\text {now }} ; i$ is the serial number of EVs; $S_{i}$ and $Q_{i}$ are the capacity and SOC of $\mathrm{EV} i$. The value of $\xi_{i}$ represents the state of the EV. If the EV is connected with the grid at time $T_{\text {now }}, \xi_{i}$ equals 1 otherwise 0 . The unit of all the capacity variables is $\mathrm{kWh}$ in this paper.

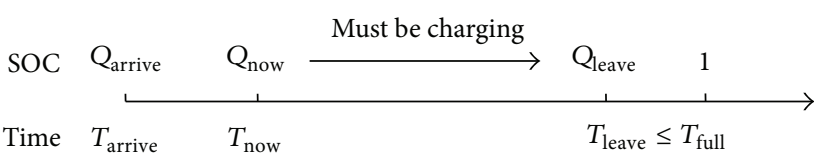

FIGURE 1: The SOC and time points of type-one EV.

However, not all the $S_{i} Q_{i}$ in (2) can be discharged for PLS. $S_{C, i}$ is used to replace $S_{i} Q_{i}$ and represent the energy which can be used. So (2) is changed into

$$
S_{\text {capacity }}=\sum_{i} \xi_{i} S_{C, i} \text {. }
$$

The different types of EV batteries have different ability to provide energy at time $T_{\text {now }}$ for load shifting. This part of energy is defined as the capacity of EVs. Actually, most of the batteries have not been used up when accessing the grid, so the capacity can be large.

2.1. Type One. If the time left is even not enough for charging the required SOC, there is no time for discharging. In this situation, the EV customer only wants to stay for a short time and then leave for another place. He hopes his car can get some energy supply at the same time. The aggregator will arrange the charging plan during all the accessing time and this EV totally becomes a load rather than energy storage. So the capacity of these EVs should be zero.

The relationship between the important time points is shown in Figure 1.

At this situation, it is defined that if $\left(T_{\text {leave }}-T_{\text {now }}\right) a<(1-$ $\left.Q_{\text {now }}\right) S_{i}$,

$$
S_{C, i}=0,
$$

where $a$ is the charging power of EV. The unit of all the charging and discharging power is $\mathrm{kW}$ in accordance with the unit of capacity. As expressed above, time parameters $T_{x}$ represent different time points. So the unit of the difference between two $T_{x}$ is $h$.

2.2. Type Two. This situation arises when there is adequate time for discharging all and then charging back. It mainly happens when people go back home and have no plan to go out for the night. In fact, most of the car users belong to this situation and their EVs constitute a large quantity of energy storage. These EVs' batteries are extremely useful for load shifting as they can be dispatched in a flexible manner. As soon as these EVs are plugged in, they can be used for load shifting and all the energy left is available. The aggregator only has to consider how to manage the energy at the peak load time. If the aggregator releases the EVs' energy too early then the energy will not be arranged at the most urgent time and the peak load will still exist. These batteries may experience several charge and discharge progress during the accessing time. But for time $T_{\text {now }}$, all the energy left in the battery is the maximum energy which can be released. 


$$
\begin{array}{r}
\text { So, if } T_{\text {leave }}-T_{\text {now }}>Q_{\text {now }} S_{i} / b+S_{i} / a \\
S_{C, i}=Q_{\text {now }} S_{i},
\end{array}
$$

where $b$ is the discharging power of EV (Figure 2).

2.3. Type Three. In this situation, charging behavior of the EV from $T_{\text {arrive }}$ to $T_{\text {now }}$ is known and do not affect the behavior after time $T$. The progress can be described as

$$
Q_{\text {now }}=Q_{\text {arrive }}+\frac{\int_{T_{\text {arrive }}}^{T_{\text {now }}} P(t) d t}{S_{i}},
$$

where $P(t)$ is the charging or discharging power of EV. When $\mathrm{EV}$ is charging, it equals $a$; when $\mathrm{EV}$ is discharging, it equals $-b$; it also can be zero when $\mathrm{EV}$ is neither charging nor discharging. However, the value of $P(t)$ is also known according to charge behavior between $T_{\text {arrive }}$ and $T_{\text {now }}$ (Figure 3 ).

At $T_{\text {now }}$, the capacity that can be used for PLS is the energy that can be discharged continuously. But after the discharge progress, the battery must be charged till full before the leaving time. In this situation, $T_{\text {stop }}$ is defined to represent the time when discharging must be stopped and charging must be started.

$$
\begin{aligned}
& \text { So, from } T_{\text {now }} \text { to } T_{\text {stop, }} \\
& \qquad S_{\text {Capacity }, i}=\int_{T_{\text {now }}}^{T_{\text {stop }}} b d t=\left(T_{\text {stop }}-T_{\text {now }}\right) b,
\end{aligned}
$$

where $b$ is the power of discharging. And at the same time, the formula is established as follows:

$$
Q_{\text {stop }}=Q_{T_{\text {now }}}-\frac{S_{\text {Capacity }, i}}{S_{i}} .
$$

Then from $T_{\text {stop }}$, it should be always in the charging mode in order to fulfill the user's request:

$$
\begin{aligned}
Q_{\text {leave }} & =Q_{\text {stop }}+\frac{1}{S_{i}} \int_{T_{\text {stop }}}^{T_{\text {leave }}} a d t \\
& =Q_{\text {stop }}+\frac{\left(T_{\text {leave }}-T_{\text {stop }}\right) a}{S_{i}} .
\end{aligned}
$$

From (9), the value of $Q_{\text {leave }}$ is also related to $T_{\text {stop. }}$. Obviously, the bigger $Q_{\text {leave }}$ is, the earlier $T_{\text {stop }}$ will be. Accordingly, $Q_{\text {stop }}$ will be smaller. It means that the grid guarantees that the batteries are always fully charged at the time when the customers plan to use the vehicles. And in this way discharge of EVs will be more acceptable. Actually, low depth charge and discharge can prolong the life of the batteries. So EVs should not be always fully charged.

With (6) (9), $T_{\text {stop }}$ and $S_{\text {capacity }, i}$ can be solved:

$$
\begin{gathered}
T_{\text {stop }}=\frac{b T_{\text {now }}+a T_{\text {leave }}-\left(1-Q_{\text {now }}\right) S_{i}}{a+b}, \\
S_{\text {Capacity }, i}=\frac{b}{a+b}\left[\left(T_{\text {leave }}-T_{\text {now }}\right) a-\left(1-Q_{\text {now }}\right) S_{i}\right] .
\end{gathered}
$$

In (10) and (11), all the variables are known at $T_{\text {now. }}$. It is feasible to evaluate the capacity and $T_{\text {stop }}$ at $T_{\text {now }}$.

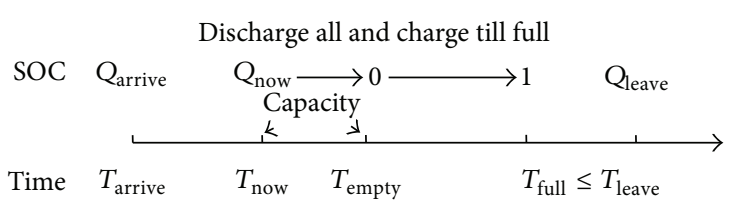

FIgURE 2: The SOC and time points of type-two EV.

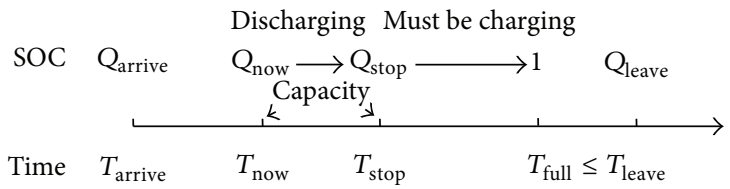

Figure 3: The SOC and time points of type-three EV.

Thus, (4), (5), (10), and (11) constitute the whole model of capacity.

\section{Capacity Evaluation and Results Analysis}

3.1. Data Illustration. References $[18,19]$ provide us a sample of different users' charging habits of EVs, such as accessing time. These habits are summarized in a probabilistic way which can be simulated based on the method of Monte Carlo. In the following study, our simulation is completely based on them and the computer software MATLAB is used to help us solve the complicated question.

Assume that all the EVs are the same and the charging and discharging power are, respectively, $5 \mathrm{~kW}$ and $4 \mathrm{~kW}$ in consideration of energy loss. And the capacity of every battery is $35 \mathrm{kWh}$.

3.2. Scenario Description. In this section, the capacity of EVs is simulated in order to know how much capacity can be dispatched for PLS. Three scenarios in [20] are set for the research under different demand side managements. The research results show how the demand side management affects the capacity. All the EVs in the simulation can charge as soon as they are plugged in. But it is assumed that the users will follow the management and try their best to pursue their benefits.

Scenario 1. Unconstrained charging: it assumes that EV charging begins as soon as the EVs access the grid and the grid can afford the load of charging.

Scenario 2. Unconstrained charging with a time delay: it assumes that EVs will delay charge for one hour or two after accessing the grid.

Scenario 3. Charging according to peak-valley time-of-use (TOU) power price: the valley time scale in this paper is from 21:00 to 8:00 in the next morning. Assume that all the users will begin charging at the valley time except for the EVs of type one. 


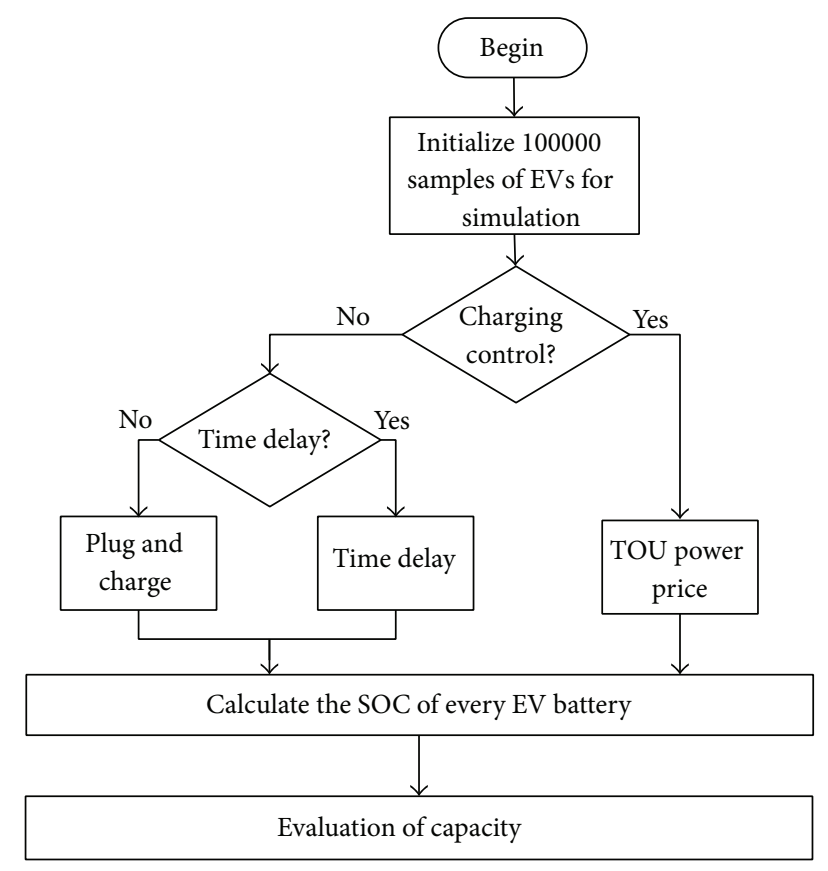

Figure 4: The flowchart of evaluation progress.

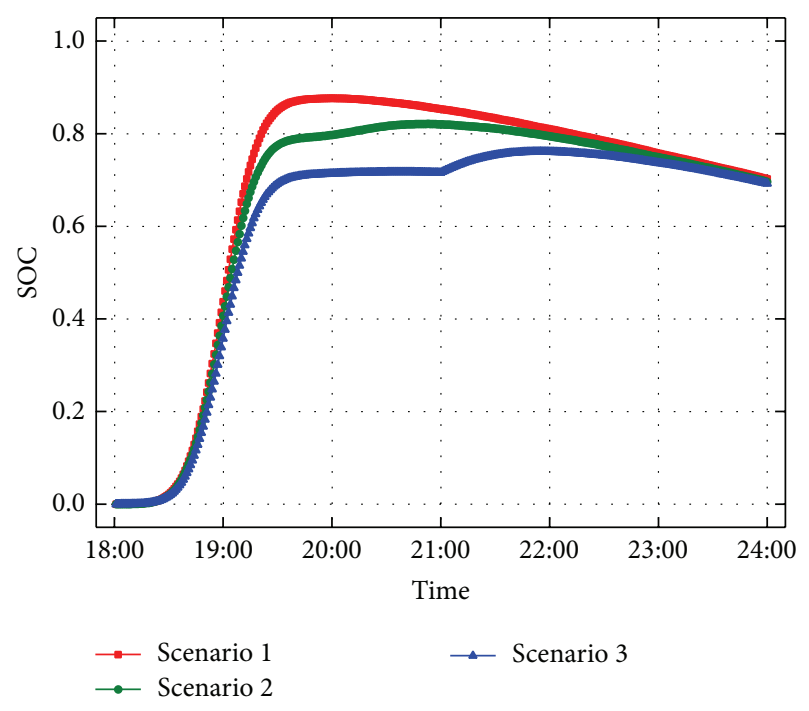

FIGURE 5: The simulation results of average SOC in three scenarios.

3.3. Simulation Results. 100000 samples of EVs are used to evaluate the average capacity. These EVs are arranged to charge under different scenarios. The specific simulation and evaluation steps are shown in Figure 4.

The simulation results from 18:00 to 24:00 are shown in the following figures. Each figure has three curves representing three scenarios. Yet, limited by the similar data samples, they follow a similar overall trend.

3.3.1. The Curves of SOC. Figure 5 shows the average SOC of all the EVs. The SOC of the EVs disconnected from the grid is recognized as 0 .

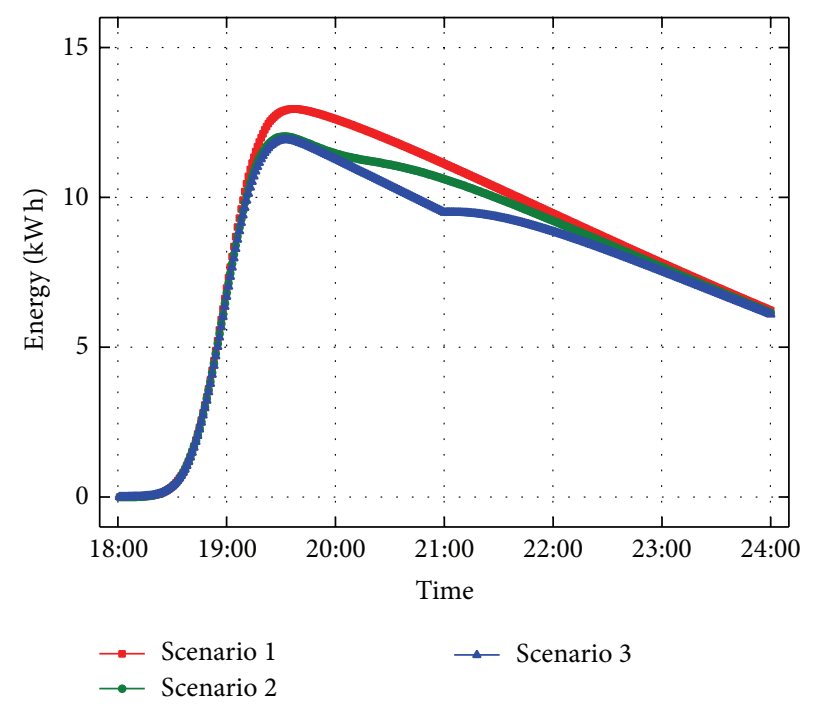

Figure 6: The simulation results of average capacity in three scenarios.

Because most of the EV users arrive home from work around 19:00, all the curves rise at first. Before 19:00, this phenomenon is dominantly caused by the accumulation of EVs rather than the growing of charging load.

After that, the EVs begin to charge in succession according to respective scenarios and express different growing patterns. Scenario 1 grows fast as the users will plug their EVs as soon as they arrive. Scenario 2 grows more gently than Scenario 1 and a part of charging load is postponed because of the time delay. In Scenario 3, one obvious plateau appears from 19:30 to 21:00. It proves that the implement of TOU power price is effective.

The peak points of SOC appear around 20:00 in Scenario 1,21:00 in Scenario 2, and 22:00 in Scenario 3. The peak points are also delayed due to the different charging strategies.

At last, the leaving of EVs leads to some energy loss. But most EVs will stay plugged-in at night which ensures the total amount of energy. Almost all the batteries in this period are fully charged.

3.3.2. The Curves of Capacity. The trend of capacity basically follows the SOC curve but it changes more gently. With the charge of EV, the capacity is enhanced. In Scenario 1, the peak points appear at similar time of SOC. But in Scenarios 2 and 3 , the peak points seem to be made earlier. Actually, it is the charging strategy that smooths those peak points which are supposed to appear later. So the demand side management strategies also benefit for the use of EVs as well as the capacity of EVs.

Figures 5 and 6 only show the results before 24:00. After 24:00, the SOC and capacity is continuously decreasing. The decrease has two reasons. Firstly, capacity calculated by formula (11) introduces that the closer the departure time is, the smaller the capacity will be. Secondly, the departure of EVs also causes the decrease of SOC and capacity. The number of EVs accessing the grid plays an important role in the capacity. 
TABLE 1: The average capacity of EVs during 18:00-24:00.

\begin{tabular}{lc}
\hline & Capacity $/ \mathrm{kWh}$ \\
\hline Scenario 1 & 8.523 \\
Scenario 2 & 8.144 \\
Scenario 3 & 7.846 \\
\hline
\end{tabular}

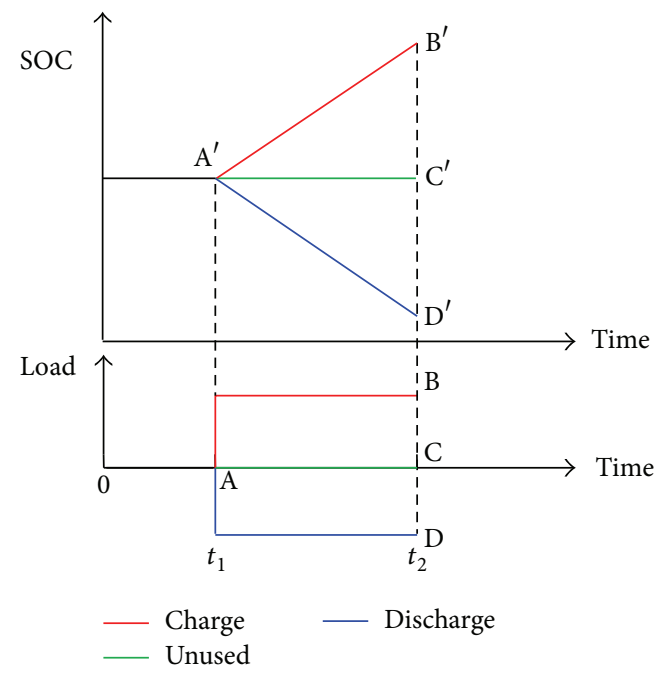

FIGURE 7: Three choices for aggregator in one period.

Table 1 shows the average discharging capacities of EVs during 18:00-24:00. The results show that each EV can provide some capacity for PLS (Figure 9). Thus, large number of EVs will form an impressive scale of capacity which can be flexibly dispatched for the grid.

\section{Peak Load Shifting with Electric Vehicles}

4.1. Electric Vehicles in One Time Period. Every time an EV accesses the grid, it has three choices for the next time period: charging, discharging, or staying unused.

If the EV chooses to charge, it will enhance the load of the grid and its SOC will rise. At the same time, the capacity which can be used for load shifting also rises.

If the EV chooses to do nothing, the state of the EV will not change in the next period. But since it remains connected with the grid, the capacity of the EV is always ready for PLS. What is more, if the unused state is changed from charging state by the aggregator, it will also lower the load of the grid just like load shedding and its effect can be remarkable.

The last choice of discharging happens mainly in the situation of PLS. The EVs are changed to be batteries' storages that can release energy to support the grid. Unlike the previous choices, discharge is a limited selection which means EV cannot choose to discharge at any time and any state. The limitation has been discussed in Section 2.

Figure 7 describes the three choices in one period between $t_{1}$ and $t_{2}$ for one EV. Different choices will bring different load to the grid. The SOC curve will also respond accordingly. The red curves show the load and SOC of charging behavior. The step value of $\mathrm{AB}$ is the slope value of

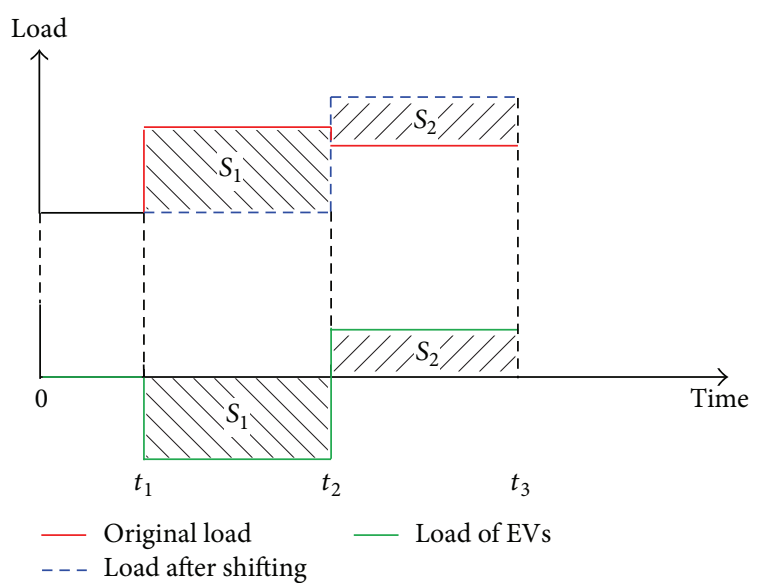

FIGURE 8: The diagram explaining how peak load shifting produces new peak load.

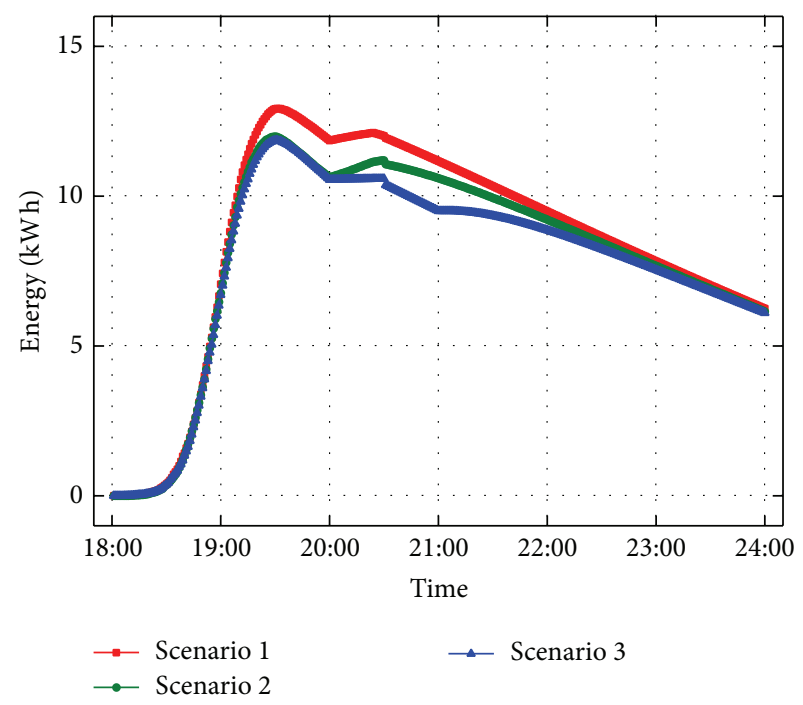

FIgURE 9: The simulation results of the capacity of EVs in PLS.

$\mathrm{A}^{\prime} \mathrm{B}^{\prime}$ which matches the power of charging. The blue curves are for discharging and the step value of $\mathrm{AD}$ (negative) is the slope value of $\mathrm{A}^{\prime} \mathrm{D}^{\prime}$ which matches the power of discharging. The curves reveal the different tendency affected on the curves on load and SOC according to different choices.

In PLS, when to use EVs for load shifting is worth considering and EVs should not be immediately dispatched at any needed time. It is because using EVs for load shifting in this period may cause EVs to be less effective in the next period. In some extreme cases, those discharged EVs must be immediately charged in the next period in order to fulfill the customers' request. In the extreme case, the discharge does not solve the peak load problem but only postpones the peak load. The result is usually to produce another new peak load which is even higher as shown in Figure 8.

In Figure 8, the red curve stands for the original load of the grid. The load is assumed to be so high that it requires load shifting at $t_{1}$. If EVs are discharging to shift the peak load of the gird in the next period $\left[t_{1}, t_{2}\right]$, the red curve can 
be lowered to be the blue curve and the result is satisfying. But in the next period $\left[t_{2}, t_{3}\right]$, part of the EVs used in $\left[t_{1}, t_{2}\right]$ requires charging. The charging load is added to the original load which in the end makes a new peak load (blue curve in $\left.\left[t_{2}, t_{3}\right]\right)$. So the PLS operation in $\left[t_{1}, t_{2}\right]$ is meaningless. The green curve describes the load of EVs in this procedure. In the energy's point of view, the EVs release the energy of $S_{1}$ in $\left[t_{1}, t_{2}\right]$ and absorb $S_{2}$ in $\left[t_{2}, t_{3}\right]$. The EVs finish an operation of transporting energy in PLS.

4.2. Electric Vehicles in Peak Load Shifting. The load of the grid is divided into several periods in the research. The peak load is the load exceeding the limitation. Usually peak load is dealing with enhancing the output of power plant. But in an emergency, the EVs can be a fantastic support. According to the request of PLS, the aggregator changes the state of a sufficient number of EVs. In this way, the load is shifted because of two reasons: the load shedding of EVs which are charging and the power produced by discharging:

$$
\Delta P=a * n_{1}+(a+b) * n_{2}+b * n_{3},
$$

where $\Delta P$ is the peak load which needs shifting; $n_{1}$ is the number of EVs whose states are changed from charging to unused. $n_{2}$ is the number of EVs whose states are changed from charging to discharging. $n_{3}$ is the number of EVs whose states are changed from unused to discharging. One group of $\left(n_{1}, n_{2}, n_{3}\right)$ constitutes a solution to the load shifting problem. So the aggregator has various solutions that can be adopted for a definite value of $\Delta L$.

However, $n_{1}, n_{2}$, and $n_{3}$ all have their value ranges according to the state before peak load. Assume that $N_{\text {charge }}$ and $N_{\text {unused }}$ are the total number of the EVs which are correspondingly charging and unused. So we have

$$
\begin{gathered}
0 \leq n_{1}+n_{2} \leq N_{\text {charge }} \\
0 \leq n_{3} \leq N_{\text {unused }}
\end{gathered}
$$

So the value range of load that can be shifted is

$$
0 \leq \Delta P \leq(a+b) * N_{\text {charge }}+b * N_{\text {unused }}
$$

As discussed in part II, what the grid needs is a long-time active power support. Equation (14) alone cannot fulfill the request. The aggregator should ensure that the capacity is also enough as

$$
\sum_{T} \Delta P \leq S_{\text {capacity }}
$$

In the progress of load shifting, the value of $S_{\text {cpacity }}$ becomes gradually smaller because of $\Delta P$. Actually, $\Delta P$ is the slope of $S_{\text {cpacity. }}$. So $\left(n_{1}, n_{2}, n_{3}\right)$ determines the $\Delta P$ and indirectly determines $S_{\text {cpacity }}$.

\section{Performance Simulation and Analysis}

To test the performance of EVs in load shifting, a simple simulation based on the scenarios in Section 3 is formed. In the simulation, almost all the EVs that can support the grid are changed into discharge by the aggregator. And the PLS that EVs take part in is from 19:30 to 20:00.

Compared with Figure 6, the capacity of EVs is reduced because of load shifting. But the curves slowly recover to their expected levels. The recovery progress is offset by the leaving EVs which makes it slower than the load shifting progress. Figure 6 proves that there will be adequate capacity left after all the available EVs have taken part in the PLS. It proves that $\mathrm{EV}$ is one kind of energy storage just like the battery storage.

\section{Conclusion}

This paper researches on the model of EVs' capacity and its performance in PLS. The three types of EVs include all the situations that the aggregator may deal with. They constitute the whole model of capacity. The model can help the aggregator evaluate the capacity before making decisions. The evaluation varies under different scenarios and shows different characteristics. The evaluation results indicate that EVs can provide potential energy to participate in PLS services.

After the simulation of capacity, we research on the principle when EVs take part in PLS. The performance is analyzed from one period to the whole PLS progress. At last, a simulation of PLS with EVs is also proposed. The result indicates that participating in PLS will not produce a harmful effect on the regular EVs' energy supply.

All the results in this paper are reasonable and meaningful for the application of PLS with EVs. What is more, the model and principle can also be referenced for other affiliation services of V2G.

\section{Conflict of Interests}

The authors declare that there is no conflict of interests regarding the publication of this paper.

\section{Acknowledgments}

This research is sponsored by National Key Technology Support Program (2013BAA01B00), National Natural Science Foundation of China (51361130152, 51377021), National Energy Administration (NY20110702-1), and Qing Lan Project.

\section{References}

[1] L. Yujun, X. Qingshan, C. Kai, and X. Xiaohui, "Prospective analysis of distribution network reconstruction on electric vehicles access to demonstration district," Elektronika ir Elektrotechnika, vol. 19, no. 6, pp. 13-18, 2013.

[2] F. Querini, S. Dagostino, S. Morel, and P. Rousseaux, "Greenhouse gas emissions of electric vehicles associated with wind and photovoltaic electricity," Energy Procedia, vol. 20, pp. 391401, 2012.

[3] J. G. Vlachogiannis, "Probabilistic constrained load flow considering integration of wind power generation and electric 
vehicles," IEEE Transactions on Power Systems, vol. 24, no. 4, pp. 1808-1817, 2009.

[4] E. Larsen, D. K. Chandrashekhara, and J. Østergård, "Electric vehicles for improved operation of power systems with high wind power penetration," in Proceedings of the IEEE Energy 2030 Conference (ENERGY'08), pp. 1-6, Atlanta, Ga, USA, November 2008.

[5] L. Xin, L. A. C. Lopes, and S. S. Williamson, "On the suitability of plug-in hybrid electric vehicle (PHEV) charging infrastructures based on wind and solar energy," in Proceedings of the IEEE Power \& Energy Society General Meeting (PES '09), pp. 1-8, Calgary, Canada, 2009.

[6] C. K. Ekman, "On the synergy between large electric vehicle fleet and high wind penetration: an analysis of the Danish case," Renewable Energy, vol. 36, no. 2, pp. 546-553, 2011.

[7] F. K. Tuffner and M. Kintner-Meyer, "Using electric vehicles to mitigate imbalance requirements associated with an increased penetration of wind generation," in Proceedings of the IEEE Power and Energy Society General Meeting, pp. 1-8, San Diego, Calif, USA, July 2011.

[8] K. Shimizu, T. Masuta, Y. Ota, and A. Yokoyama, "Load Frequency Control in power system using Vehicle-to-Grid system considering the customer convenience of Electric Vehicles," in Proceedings of the International Conference on Power System Technology (POWERCON '10), pp. 1-8, Hangzhou, China, October 2010.

[9] S. B. Peterson, J. F. Whitacre, and J. Apt, "The economics of using plug-in hybrid electric vehicle battery packs for grid storage," Journal of Power Sources, vol. 195, no. 8, pp. 2377-2384, 2010.

[10] J. Taylor, A. Maitra, M. Alexander, D. Brooks, and M. Duvall, "Evaluation of the impact of plug-in electric vehicle loading on distribution system operations," in Proceedings of the IEEE Power and Energy Society General Meeting (PES '09), pp. 1-6, Calgary, Canada, July 2009.

[11] L. Jian, H. Xue, G. Xu, X. Zhu, D. Zhao, and Z. Y. Shao, "Regulated charging of plug-in hybrid electric vehicles for minimizing load variance in household smart microgrid," IEEE Transactions on Industrial Electronics, vol. 60, no. 8, pp. 32183226, 2013.

[12] M. Datta and T. Senjyu, "Fuzzy control of distributed PV inverters/energy storage systems/electric vehicles for frequency regulation in a large power system," IEEE Transactions on Smart Grid, vol. 4, no. 1, pp. 479-488, 2013.

[13] J. Fluhr, K. Ahlert, and C. Weinhardt, "A stochastic model for simulating the availability of electric vehicles for services to the power grid," in Proceeding of the 43rd Annual Hawaii International Conference on System Sciences (HICSS '10), pp. 110, Honolulu, Hawaii, USA, January 2010.

[14] S. Han, S. Han, and K. Sezaki, "Estimation of achievable power capacity from plug-in electric vehicles for V2G frequency regulation: Case studies for market participation," IEEE Transactions on Smart Grid, vol. 2, no. 4, pp. 632-641, 2011.

[15] M. Takagi, H. Yamamoto, and K. Yamaji, "Evaluation of expanded allowable capacity of wind power in power systems by charge control for plug-in hybrid electric vehicles," Electrical Engineering in Japan, vol. 188, pp. 39-48, 2012.

[16] A. Oudalov, R. Cherkaoui, and A. Beguin, "Sizing and optimal operation of battery energy storage system for peak shaving application," in Proceedings of the IEEE Lausanne Power Tech, pp. 621-625, 2008.

[17] S. G. Tesfahunegn, Ø. Ulleberg, P. J. S. Vie, and T. M. Undeland, "Optimal shifting of Photovoltaic and load fluctuations from fuel cell and electrolyzer to lead acid battery in a Photovoltaic/hydrogen standalone power system for improved performance and life time," Journal of Power Sources, vol. 196, no. 23, pp. 10401-10414, 2011.

[18] Z. Luo, Z. Hu, Y. Song, X. Yang, K. Zhan, and J. Wu, "Study on plug-in electric vehicles charging load calculating," Automation of Electric Power Systems, vol. 35, no. 14, pp. 36-42, 2011.

[19] J. Taylor, A. Maitra, M. Alexander, D. Brooks, and M. Duvall, "Evaluations of plug-in electric vehicle distribution system impacts," in Proceedings of the IEEE Power and Energy Society General Meeting (PES '10), pp. 1-6, Minneapolis, Minn, USA, July 2010.

[20] J. Wang, C. Liu, D. Ton, Y. Zhou, J. Kim, and A. Vyas, "Impact of plug-in hybrid electric vehicles on power systems with demand response and wind power," Energy Policy, vol. 39, no. 7, pp. 40164021, 2011. 


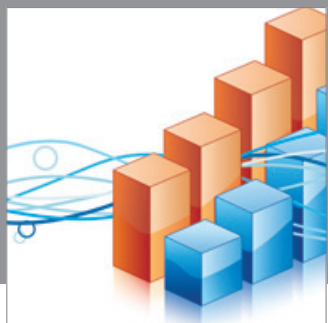

Advances in

Operations Research

mansans

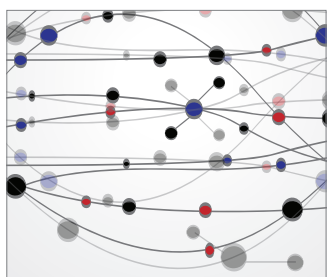

The Scientific World Journal
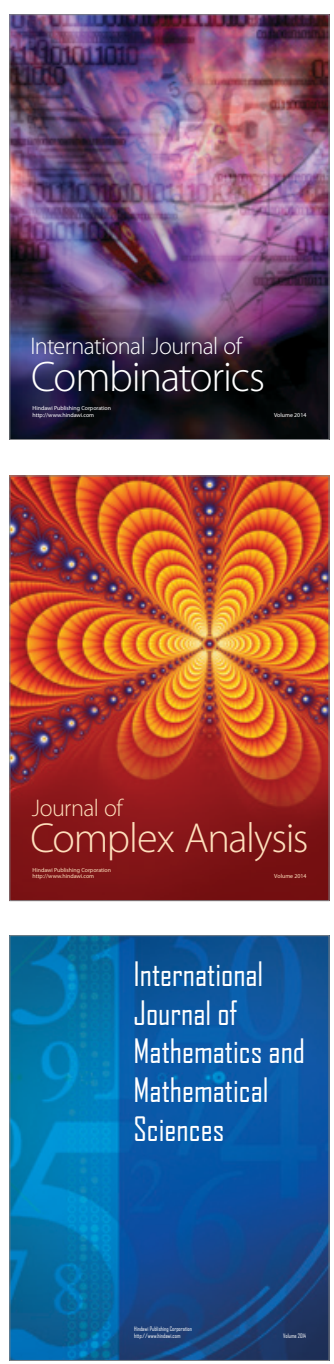
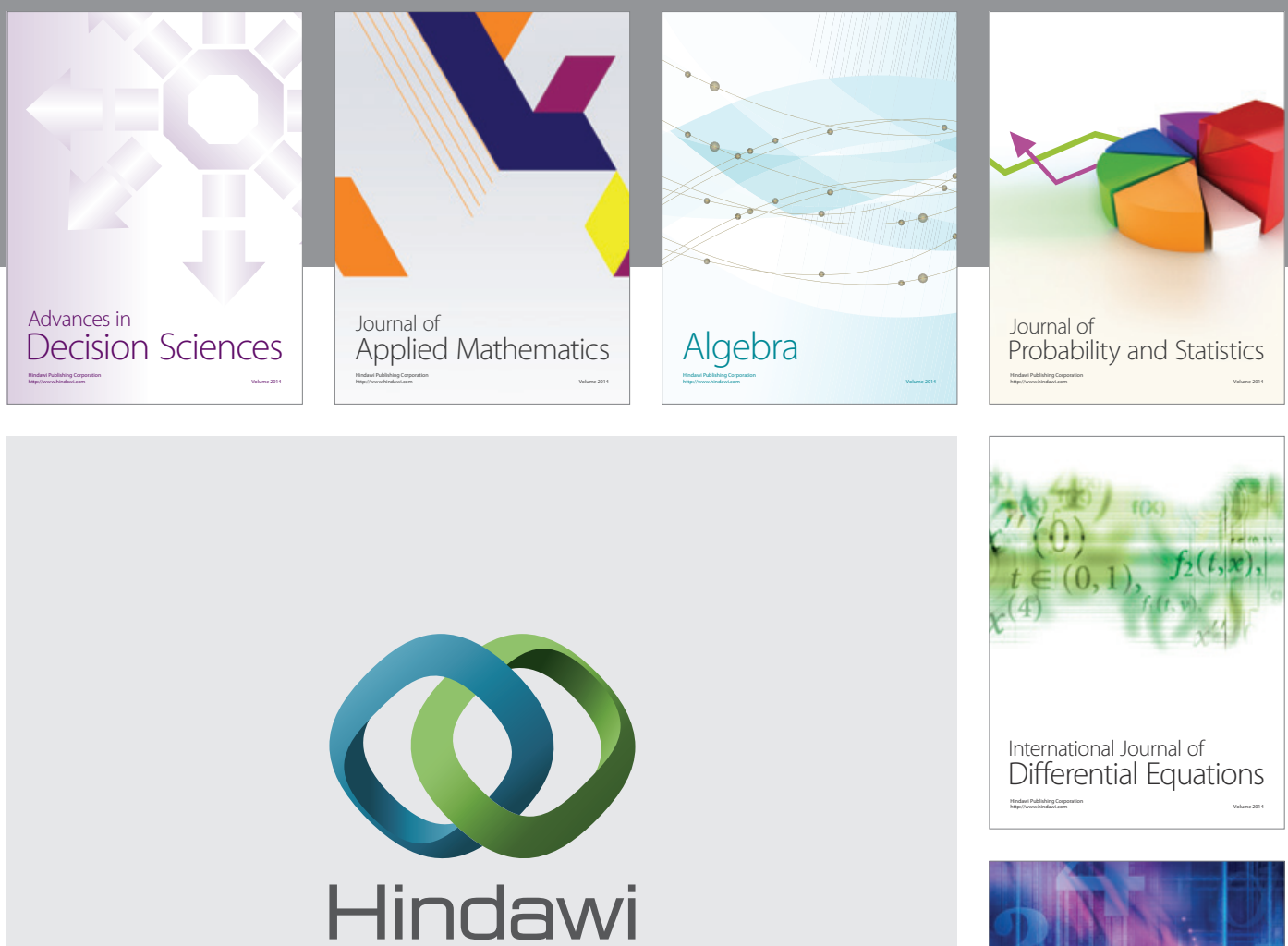

Submit your manuscripts at http://www.hindawi.com
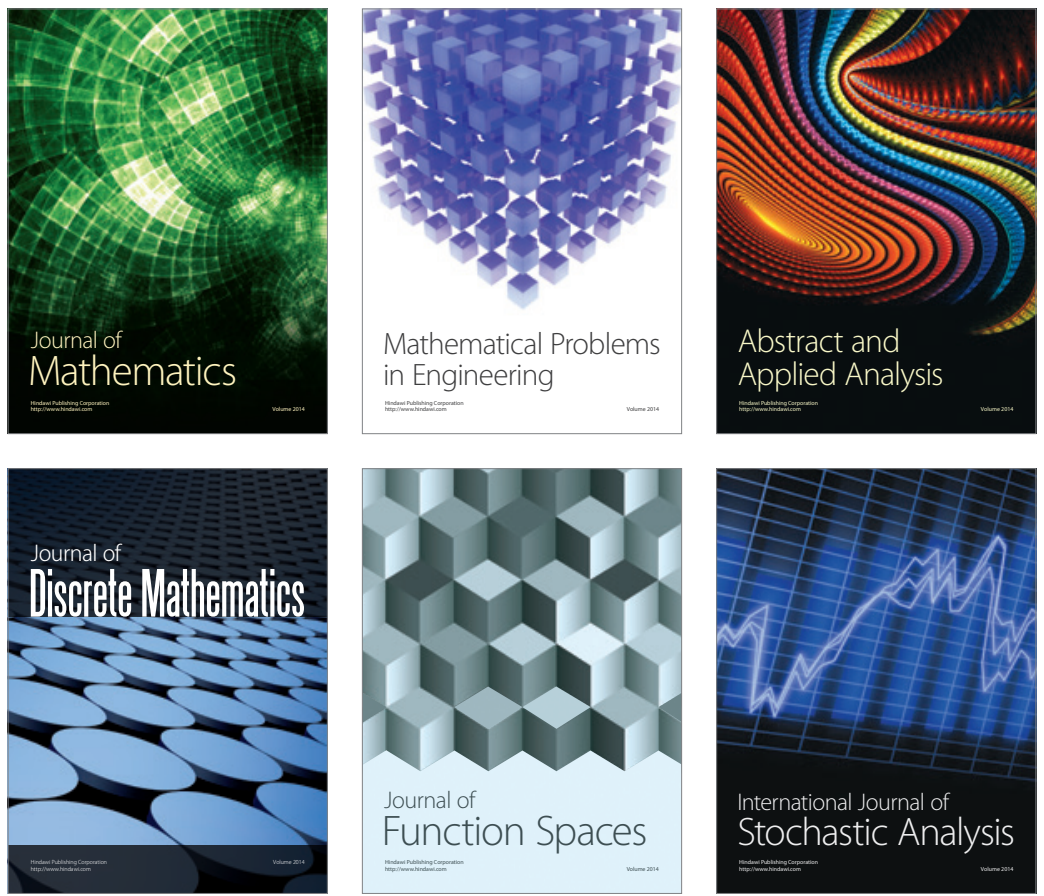

Journal of

Function Spaces

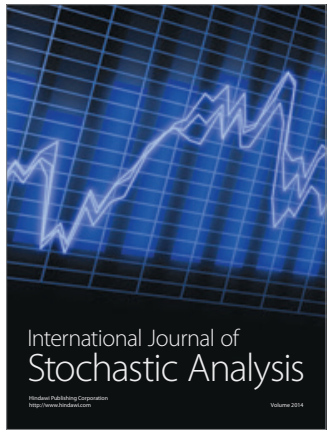

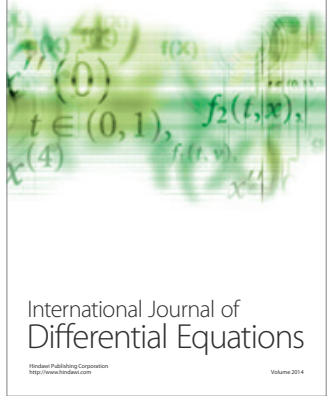
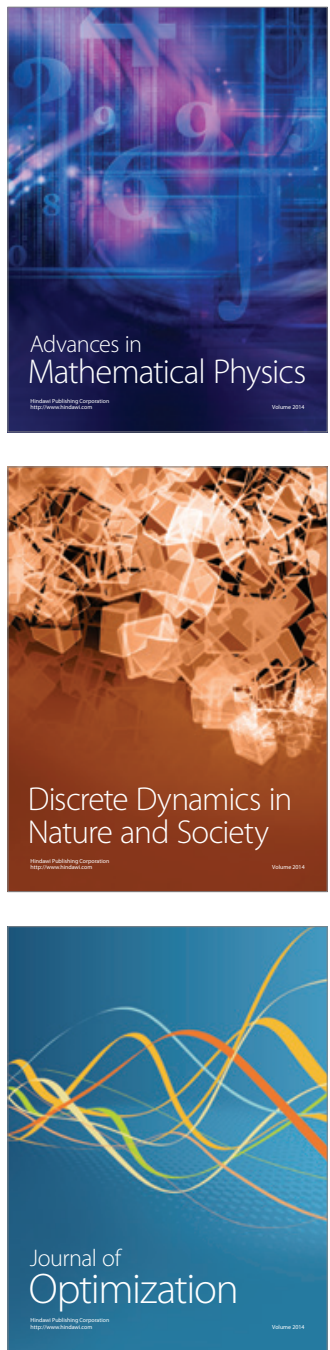